\title{
Gewaltverortung und Gewaltkodierung. Zu den Berlin-Romanen von Inka Parei, Ingo Schramm und Tim Staffel
}

\begin{abstract}
Niniejszy artykuł koncentruje się na ukazaniu problemu kodowania przemocy, wyrażonej w powieściach o Berlinie autorstwa Inki Parei, Ingo Schramma i Timma Staffela oraz podejmuje próbę odpowiedzi na pytanie o formy i skutki przemocy dotykające postaci literackich oraz o związek przemocy z literackim toposem Berlina jako metropolii. Wszyscy trzej autorzy ukazują stolicę Niemiec jako siedlisko różnego rodzaju przestępstw i głęboko zaniedbane miejsce - zarówno pod względem zewnętrznym, jak i wewnętrznym.
\end{abstract}

Im folgenden Beitrag wird die Gewaltkodierung in den Berlin-Romanen von Inka Parei, Ingo Schramm und Timm Staffel untersucht. Betrachtet werden die unterschiedlichen Gewaltformen, das Wirken von Gewalt auf die Romanfiguren sowie der Zusammenhang zwischen der Gewaltthematik und dem literarischen Topos ,Großstadt Berlin'. Die drei Autoren schildern die deutsche Hauptstadt als Ort des Verbrechens und tiefster äußerer und innerer Verwahrlosung.

This article deals with the codification of violence in the Berlin novels of Inka Parei, Ingo Schramm and Timm Staffel. The main focus lies on forms of violence and the impact of violence on the novels' heroes as well as on the connectivity of violence with the literary place of the Berlin metropolis. The three authors portray the German capital as a place of crime and profound internal and external decay.

Keinem, der dem Wesen der menschlichen Angelegenheiten, das sich in Geschichte und Politik manifestiert, nachdenkt, kann die Rolle, welche die Gewalt seit eh und je in den Beziehungen der Menschen zueinander gespielt 
Aleksandra Chylewska-Tölle

hat, entgehen; und es ist auf den ersten Blick einigermaßen überraschend, daß sie so selten zum Gegenstand besonderer Untersuchungen gemacht wurde ${ }^{1}$,

konstatiert HANNAH ARENDT (1971:12) in ihrer Studie Macht und Gewalt. In diesem Sinne wird sich der vorliegende Artikel mit dem Phänomen der Gewalt aus literarischer Sicht auseinandersetzen. Betrachtet werden dazu drei Berlin-Romane einer jüngeren Autorengeneration (geboren in den 1960er Jahren), die in der literarischen Öffentlichkeit ein hohes Maß an Aufmerksamkeit erregen konnten. Nachgegangen werden soll der Frage, wie Gewalt in diesen Romanen verstanden und dargestellt wird. Analysiert wird dabei ebenso die Kodierung von Gewalt im Sinne ihrer literarischen Darstellung wie die sichtbare oder verdeckte Wirkung von Gewalt auf die Romanfiguren. Es wird dabei nötig sein, eine Gewalt, die nach Nietzsche als kreativ zu betrachten ist und eine Palette von Möglichkeiten für die positive Entwicklung des Menschen eröffnet, von einer Gewaltform zu unterscheiden, die sich destruktiv auf das menschliche Leben auswirkt. Betrachtet wird Gewalt sowohl aus der Opfer- als auch aus der Täterperspektive. Zudem sind unterschiedliche Gewaltmotive zu berücksichtigen wie Schuld, Zukunftsangst, Identitätssuche oder versuchter Neuanfang. Ein zusätzliches Kriterium bei der Auswahl der Texte ist der Handlungsort Berlin. Gefragt wird daher u. a. danach, wie das Gewaltmotiv mit dem literarischen Topos ,Großstadt Berlin verknüpft ist, d.h. wie die deutsche Hauptstadt nach dem Mauerfall 1989 als Ort einer ubiquitären Aggressivität inszeniert wird. Berlin ist bei Parei, Schramm und Staffel keineswegs ein zufälliger Schauplatz; ihre Romane zielen auf die Stadt selbst, der bei der Verbreitung von Gewalt eine bestimmte Rolle zugeschrieben wird. Es wäre somit auch zu fragen, inwiefern die drei Texte im weiteren Sinne Rezeptionsgewohnheiten der Leser destruieren.

Der im Jahre 1999 veröffentlichte Roman Die Schattenboxerin von Inka Parei fasst die alles zerstörende Gewalt hauptsächlich in einer Metaphorik. Der aus Westdeutschland stammenden Autorin, die seit den 1990er Jahren in Berlin lebt, geht es vorrangig um die Darstellung der Überlebensstrategien einer vergewaltigten Frau namens Hell, die sich durch den Rückzug ins Private etlichen Zwängen der Gesellschaft zu entziehen versucht:

Ich stöpsele das Telefon aus, beantrage Waisenrente, vermeide Außenkontakte. [...] An manchen Tagen scheint mein Leben von der Zimmertür bis zur Balkonbrüstung zu reichen, an anderen nur bis zu den Kanten der Matratze. Hin und

1 Hannah Arendt führt eine reichhaltige Literatur über Krieg und Kriegführung an, die sich jedoch ausschließlich auf Gewaltmittel und nicht auf Gewalt als solche bezieht. 
wieder endet es an der Stelle, wo mein Körper seine psychische Ausdehnung erreicht hat. Ich ahne, dass es vielleicht möglich wäre, sich noch weiter zu minimieren, nach Innen hinein, aber an diesem Punkt überfällt mich Angst, und ich stehe auf. Ich ziehe mich an, leere den Nachttopf, der neben dem unbenutzten Stromheizer steht, zwinge mich auf die Straße hinaus, in die Grünanlagen am Kanal, koche gelegentlich, mache Ordnung, beginne meinen Rückzug erneut. (PAREI 2001:113f.)

Die Protagonistin kennt weder Stabilität noch das Gefühl der Sicherheit; sie erscheint sowohl als Opfer ihres Geschlechtes als auch der Gesellschaft im Allgemeinen. Ihre Beobachtungsgabe erlaubt eine eindringliche Analyse ihrer Umgebung, wobei des Öfteren eine verzerrte Wahrnehmung zu bemerken ist. In ihren Phantasien und immer neu durchlittenen Alpträumen sieht Hell die überall auf sie lauernden Gefahren, und ihre Ängste spiegeln sich in zahlreichen inneren Monologen: „Weglaufen ist mein erster Impuls. Weglaufen ist nicht nur ein legitimes, sondern in ausweglosen Situationen überhaupt das beste Mittel. Die letzte der Strategien. Die man anwendet, wenn alle anderen versagen." (PAREI 2001:60)

Die Autorin vermeidet in ihrem Roman eine tendenziöse, moralische Aussage, die einzelnen Geschehnisse werden gelassen, ohne Pathos, still, metaphorisch verschlüsselt und voll von unheimlicher Spannung erzählt. Die von Parei gewählte Form des inneren Monologs vermittelt einen Entwicklungsprozess der Wahrnehmung, der sich anhand der fast ausschließlich gewählten Ich-Perspektive präzise verfolgen lässt. Nicht zu übersehen ist darüber hinaus der Verzicht auf explizite Kommentare, deutende Erklärungen und abschlieBende Interpretationen des Gezeigten. Die Autorin bedient sich vielmehr zahlreicher Chiffren, benutzt Metaphern und Gedankenkürzel, die beim Leser unterschiedliche Assoziationen wecken. Diese Technik der Andeutung und des Aussparens resultiert aus ihrem Konzept, eine entstellte Widerspiegelung der Realität darzustellen.

Wie sich gleich anhand der ersten Szene des Romans belegen lässt, generiert die Autorin ein Höchstmaß an Konzentration, indem sie eine gedrängte Fülle von Einzelheiten schildert. Die von der Protagonistin reflektierte eigene Zeiterfahrung macht den Leser auf die Dualität der Handlungszeit aufmerksam. Hell lebt zurückgezogen in ihrer eigenen Zeit, aber zugleich erlebt sie die Berliner Wende- und Nachwendejahre. Die Konstruktionselemente des Romans bleiben einer flüchtigen Lektüre verborgen und enthüllen sich erst durch detektivisches Aufspüren. Die facettenreiche Verknüpfung von Alltagserfahrungen und deren Bewältigung mit dem Problem des inneren Zeitbewusstseins bilden ein einzigartiges Mosaik, wobei jede Kombination 
Aleksandra Chylewska-Tölle

der Koordinaten wie insbesondere die Lichtmetaphorik, d.h. die Betonung des Gegensatzes zwischen Helligkeit und Dunkelheit (Dunkel heißt die vermisste Nachbarin der Protagonistin), einen neuen Schlüssel zum Textverständnis liefert. Die unterschiedlichen Bilder lassen sich daher nicht isoliert voneinander, sondern erst durch die Analyse ihrer spezifischen Aufeinanderbezogenheit innerhalb des Textes vollständig verstehen.

Parei nutzt die Stadt als Schlüssel zur Erkundung von Hells Identität. Dadurch wird die Darstellung Berlins als Reflexion des subjektiven Erlebens auf das Subjekt zurückgelenkt. Die deutsche Hauptstadt wird in dieser Konstellation zu einem Zeugen von Hells Gewaltverarbeitung. So wie sich die Stadt nach dem Mauerfall drastisch veränderte, so verändert ist auch die Wahrnehmung der Stadt seitens der Protagonistin als Folge ihrer Vergewaltigung. So transzendiert ihre Sprache über sich selbst, über die künstlerische Setzung des Wortes hinaus: als Aussage einer Seinserfahrung, als Weltbekenntnis, als Bezeugung der Wahrheit. Eine wichtige Funktion im Roman nehmen die Verweise auf die Weltmetropole an der Spree ein, die als grau, dunkel und zerfallen erscheint.

Ingo Schramms Fitchers Blau (1996) ist als einziger der hier behandelten Werke als ,Wenderoman' zu bezeichnen. Es besteht nicht nur ein zeitlicher Zusammenhang mit der Wende 1989/1990, sondern - was wichtiger ist - die Wende hat zudem im Unterschied zur Schattenboxerin einen unmittelbaren Bezug zur Handlung. Neben dem in diesem Roman behandelten Diskurs über die Folgen von Gewaltanwendung findet sich eine politische Dimension in Form einer Verflechtung mit historischen Ereignissen, die das Europa der Nachkriegszeit nachhaltig geprägt haben und im Fall des Eisernen Vorhangs gipfeln. Die Geschichte selbst ist jedoch im Roman nur in undeutlichen Umrissen präsent und oszilliert wie in vielen Werken ostdeutscher Autoren nach 1989 zwischen den Polen Abschied und Anfang (KORMANN 1999:121). Was zunächst nur angedeutet wird (wie z.B. die Situation vieler Ost-Berliner nach der Wiedervereinigung), wird später aus einer anderen Perspektive gezeigt und durch zusätzliche Ereignisse verstärkt. Erzählt wird die Geschichte der zwei 25jährigen Ostdeutschen Karl Klemm und Janni Mavigogova, die was sich erst in der Endphase des Romans zeigt - Halbgeschwister sind. Beide versuchen, im Nachwende-Berlin ihren Platz zu finden. Diese Suche wird von vielen Enttäuschungen begleitet, weil die anfängliche Begeisterung nach der Wiedervereinigung schnell einer bitteren Ernüchterung weicht. Beim Aufeinandertreffen von Ost und West kommt es zu Konfrontationen und Problemen, die das Ausmaß der deutsch-deutschen Entfremdung deutlich 
zeigen; Karls Niederlagen bei der Suche nach einem Job sind „klassische Wendeerfahrungen“" (STENGER 1997:17). Die eigentliche Hauptfigur des Romans ist jedoch Josef, der Vater von Karl und Janni, dessen Lebensgeschichte von Aggressivität und Gewalt geprägt ist und mit vielen Einzelheiten vom auktorialen Erzähler geschildert wird. An seinem Schicksal können die Aspekte der Metaphorisierung des Gewaltphänomens gezeigt werden. Josef lässt sich darüber hinaus als ein besonderer Fall von kollektiver Verdrängung der deutschen Geschichte betrachten. Der hochangesehene Arzt in der Nationalen Volksarmee wurde in seiner Kindheit oft verlassen, misshandelt, erniedrigt und zusammengeschlagen. Vor allem im Kinderheim sind ihm auf Schritt und Tritt Hohn und Spott begegnet. Von klein auf hat er erfahren, dass nur der Stärkere überlebt, und demgemäß hat er seine Lebensstrategie entwickelt: Gewalt muss immer neue Aggressivität und infolgedessen neue Gewalt ${ }^{2}$ zeugen. Sein ständiger Kampf als ,inneres Erlebnis، erscheint hier als wüster, alles Menschliche vernichtender Fiebertraum. Die Härte gegenüber seinen am gleichen Tag von zwei Frauen geborenen Kindern und seine Neigung, andere Menschen in seiner Umgebung anzugreifen und zu verletzen, hat seine Ursache in Josefs Kriegserfahrung und in seinem Werdegang in der stalinistisch geprägten DDR. Selbst täglich mit Gewalt konfrontiert, überträgt er sie auf andere, vor allem in Form einer Strafe, die zum Ziel hat, Unterordnung, Gehorsam und Disziplin zu erzielen. Mit anderen Worten: Josef wendet Gewalt als soziotechnisches Mittel an und setzt in der Erziehung seiner Kinder Angstreize in der Überzeugung ein, dass diese stimulierend wirken und zur Herausbildung gewisser Haltungen beitragen:

Strafe musste wie aus dem Nichts einschlagen. Aus dem Ungewissen heraus. Vollstreckt in der unschuldigsten Sekunde des Lebens. Wenn der Schuldige schon vergessen hatte, dass er die schwerste Schuld trug. Die Strafe begründete auch die Schuld. Wer bestraft wurde, hatte Unrecht auf seine Seite gezogen. Strafe war immer im Recht. Denn nur der Mächtige konnte strafen. Niemand, der nicht mächtig, war zu einem anderen Sinn, als bestraft zu sein. Es ging um die Begründung der Strafe. Die Strafe hatte erhalten zu werden, als allgegenwärtige Drohung. Nicht nur als Drohung, auch als Vollzug. Denn wäre die Strafe verschwunden, dann wäre die Ordnung verschwunden. Ordnung war das halbe Leben. Die andere Hälfte war Recht, also Strafe. (SCHRAmm 1996:410)

Dass hier die Geschichte von Josef als Destruktion seiner Persönlichkeit und Weltanschauung erzählt wird, deutet sich schon im Titel des Romans an. Er

2 Aggressivität unterscheidet sich von Gewalt vor allem dadurch, dass sie eine Emotion ist. Gewalt hingegen manifestiert sich in Taten (WITORSKA 2005:145). 
Aleksandra Chylewska-Tölle

bezieht sich nämlich auf das Grimm'sche Märchen Fitchers Vogel, das eine Variante des Blaubart-Stoffs darstellt. Im Märchen kann der Hexenmeister, der die Frauen entführt, in einer „Blutkammer“ aufbewahrt und schließlich tötet, überlistet und schließlich verbrannt werden. Bei Schramm fehlt zwar der Märchenschluss, doch die Anspielung auf das Phänomen Gewalt in ihrem Körperbezug und in ihrem Verhältnis zur Ausübung von Macht ist hier deutlich zu bemerken.

Im Roman wird der Stadt Berlin, ähnlich wie bei Parei, eine wichtige Rolle zugeschrieben. Die Stadt tritt in Fitchers Blau als eigenständige Symbolwelt auf. Ihre Funktion als Ort der städtischen und herrschaftlichen Repräsentation, die sie vor dem Zweiten Weltkrieg innehatte, und ihre Bedeutung als Ort des beginnenden Ost-West-Antagonismus in der unmittelbaren Nachkriegszeit wird an mehreren Beispielen diskutiert. Mit anderen Worten, Berlin als Zentrum der Wende-Ereignisse ist für viele ehemalige Ost-Berliner der Nährboden für Entwurzelungsprozesse und ihnen nachfolgende gescheiterte Assimilationsversuche. Den jungen Protagonisten vermittelt ihre Heimatstadt die Grunderfahrungen ihres Daseins und erschließt ihnen die Zusammenhänge zwischen ihren Empfindungen, Träumen und Bedürfnissen. Sie vergegenwärtigt sowohl ihre Hoffnungen als auch ihre Angstvorstellungen und ist ein schweigender Zeuge der sozialgeschichtlichen Entwicklungen und psychischen Veränderungen ihrer Bewohner. Überall ist bittere Ironie zu spüren:

,Mehr Demokratie - mehr Sozialismus.' Das war weggewischt worden, übertüncht von den Wächtern der Despotie. Denn die hatten gewusst, dass so ein Satz Lüge gewesen. [...] In den Wohnungen rings lebten glückliche Deutsche, kurz vor der Nacht sehr entschlossen, zum Fernsehen bereit und stolzgeschwellt seit vier Wochen. In allem einig, ein arbeitsam glückliches Volk. (SCHRAMM 1996:21)

Die abrupt und unvollständig beendeten Sätze, die den Zerfall der überkommenen Werte und die Auflösung der traditionellen Bindungen anzeigen, sind auch als eine Form der narrativen Provokation anzusehen. Sie symbolisieren die Krisensituation als Übergang vom Alten zum Neuen. Diese manifestiert sich zudem in der retrospektiven Betrachtung der deutschen Vergangenheit, wobei jedoch bei Schramm von einer ,Ostalgie' kaum die Rede sein kann. Der Autor zielt vielmehr auf die Darstellung von Berlin im Wandel und stellt das Fehlen der notwendigen, raschen Anpassung der Gesellschaft an die neuen Verhältnisse dar.

Dass Gewalt ansteckend ist, zeigt auch Timm Staffel in seinem Roman Terrordrom (1998). Die von ihm dargestellte Bedrohung ist ein grundsätzliches 
Kennzeichen des Lebens, und die Gewalt selbst wird vor allem als Instrument der Machtdemonstration begriffen. Die Zeitspanne der Handlung erstreckt sich von Ende November 1999 bis August 2000, sie umfasst also symbolisch die Schwelle zum 21. Jhd. Obwohl die Begebenheiten im realen Berlin stattfinden, weist Staffels Geschichte irreale und sogar surrealistische Züge auf. Erzählt wird von den Folgen einer neuen Eiszeit, in der die ganze Infrastruktur der Stadt zusammenbricht: Eine unvorstellbare Kälte verursacht, dass Abwasserrohre platzen, große Schneemassen anwachsen, der Straßenverkehr ins Stocken gerät und ganze Stadtviertel evakuiert werden müssen. Der Frost bedeutet den Untergang einer Ordnung, die trotz ihrer scheinbaren Unerschütterlichkeit vom totalen Zerfall erfasst wird. Einer der Auslöser ist Lars, der anonym seine nihilistischen Manifeste, die nur mit dem Buchstaben , $\mathrm{V}^{*}$ unterschrieben sind, überall verteilt. In den Manifesten wird der Rechtsstaat auf höhnische Weise in Zweifel gezogen; in einem von ihnen ist zu lesen:

Eine Gewalttat ist immer ein Unfall. Die Frage ist, warum die Gewalttat immer auf den einzelnen bezogen wird oder auch eine Gruppierung, die sich von der Mehrheit unterscheidet durch eben diese Gewalttat. Denn diese Gesellschaft ist nicht gewalttätig. Die Menschen haben gelernt, die Rolle des Opfers zu übernehmen, und auf der Suche nach den Tätern ermitteln sie die Minderheiten. Und wird die Minderheit Opfer eines Unfalls, so hat sie ihn natürlich selbst verursacht. Die Verantwortlichkeit eines ,,andersartigen“ ist sehr viel höher angesetzt als die der Mehrheit. (STAFFEL 1998:41)

Zuerst werden diese Hassbriefe kaum wahrgenommen. Mit der Zeit verbreitet sich aber in der Stadt das Gefühl eines permanenten Schreckens, weil die Formen und die Dynamik der Gewaltakte dramatisch zunehmen. Sie erschöpfen sich nicht nur in der Zerstörung von Gebäuden, der Vergewaltigung und Verletzung von Personen, sondern führen zu Morden und Selbstmorden. Es mehren sich darüber hinaus sorgfältig geplante terroristische Anschläge, die viele Parallelen zum sozialrevolutionären Terrorismus aufweisen, insofern dieser grundlegende Veränderungen in Stadt und Gesellschaft anstrebt (IMBUSCH 2006:494). Dadurch dass über die multimediale Vernetzung Gewalttaten an entfernt liegenden Orten zum Bestandteil der alltäglichen Information werden, herrscht in der Stadt ein Bewusstsein permanenter Bedrohung und Brüchigkeit des menschlichen Lebens. Die Wetterverhältnisse und die Gewaltexzesse gehen einher mit tiefgreifenden Veränderungen in der Gestaltung des Alltagslebens der Berliner Bevölkerung. Die einen werden durch die Vorahnung einer nahen apokalyptischen Katastrophe, die sich in einigen prophetischen Stimmen äußert, gelähmt. Eschatologische Gedanken gehen bei ihnen jedoch nicht einher mit der Bemühung um eine sinnvolle Gestaltung des Lebens und mit Sehnsucht nach Harmonie. Die anderen versuchen, 
aus diesem Endzeitbewusstsein den größtmöglichen Nutzen zu ziehen. $\mathrm{Zu}$ dieser Gruppe gehört der Fernsehmoderator Tom, der die Erschütterungen für seine Talkshows nutzt. Seine geschickte Marketingstrategie führt zur Gründung eines Vergnügungsparks für konsumierbare Gewalt, die als großer Erfolg live übertragen wird:

Wir organisieren den allgemeinen Terror selbst. Wir reglementieren ihn. Wir verdienen damit Geld. Das ist es. Und niemand wird unfreiwillig verletzt. Alles auf eigene Gefahr. [...] Wir erklären ein riesiges, abgesperrtes Areal zum Kriegsgebiet, in dem sich jeder nach seinen Wünschen und Überzeugungen austoben kann. Ständige Übertragung von sämtlichen Aktivitäten jeder Gruppierung. Eine riesige Arena. Ein riesiger Schaukampf. Jeder gegen jeden. Alles offiziell: Gegner, Waffenausgabe, Trainingslager. Dasselbe wie jetzt, nur offiziell und mit der Gewähr, dass es sich nicht totläuft. V errichtet sein Terrordrom. Seinen Staat im Staat. Und alle glauben ihm das und spielen mit. Eine gigantische Spielothek. (STAFFEL 1998:189)

Staffel etabliert den Handlungsort Berlin als Kollektivsymbol, d.h. die Stadt wird zu einer Stätte, die von Endzeitstimmung und apokalyptischen Visionen durchtränkt ist, verbunden mit Gefühlen der Verunsicherung und Entfremdung ihrer Bewohner. Die Spreemetropole wird dabei möglichst authentisch gestaltet, was u.a. unzählige, genaue Ortsangaben und eine einfache und realitätsnahe Sprache des Romans belegen. Doch Staffels Berlin steht in einem auffälligen Kontrast zur jüngeren deutschen Geschichte, die ihren Neuanfang mit dem ruhmreichen Mauerfall genommen hat. Jetzt, zehn Jahre nach diesen Geschehnissen, ist der Mensch nicht mehr fähig, den Lauf der Geschichte zu steuern, geschweige denn ihn auch nur geistig nachzuvollziehen.

Obwohl Nihilismus, Verzweiflung und groteske Entfremdung bei allen drei Autoren überaus präsent sind, formieren diese Aspekte nicht die letztgültige Aussageschicht ihrer Werke. Allen drei Romanen gemeinsam ist das Bewusstsein einer totalen Zeit, einer unabänderlichen Zeitenwende. Die Romanfiguren wachsen auf in Zeiten „permanenter Gewaltpräsenz“ (REEMTSMA 2008:55). Alle drei Autoren integrieren die Gewaltthematik in die Alltagserfahrung, wobei die Bewertung des Gewaltphänomens sich in vielerlei Hinsicht einer oftmals gewünschten Eindeutigkeit entzieht. Dies resultiert u. a. daraus, dass ,Gewalt ‘ von den Autoren aus unterschiedlichen Perspektiven beleuchtet wird. Parei schildert die intime und allzu vielen Frauen bekannte Existenzproblematik ${ }^{3}$, die sich bei ihrer Protagonistin durch

3 Laut einer wissenschaftlichen Studie hat jede siebte Frau in Deutschland nach dem 16. Lebensjahr sexuelle Gewalt erlebt (MüLLER / SCHRÖTTLE 2006:81). 
Gefühlsgehemmtheit und Berührungsscheu als Folge einer Vergewaltigung manifestiert. Von eben diesem Erlebnis her, durch das ihr Leben zusammengebrochen ist, müssen alle späteren Schilderungen gelesen werden, die manche Details über Hells Bewältigungsstrategien enthüllen. Sie lernt asiatische Verteidigungskunst, gewinnt ein distanziertes Verhältnis zum Leben und ergreift in ausweglosen Situationen die Flucht. Sie schlendert nach dem traumatischen Erlebnis ziellos durch die Stadt, wobei mit der Zeit der Radius ihrer Wanderungen durch Bahnfahrten erweitert wird. Die erfahrene Aggressivität entwickelt bei der Titelfigur ein hochsensibles Bewusstsein von den Grenzen der Sprache, d.h. von einer Differenz zwischen Reden, Schweigen und Verschweigen. Nur durch ihre inneren Monologe seziert sie ihre Umgebung. Die Monologe dienen der Stabilisierung ihrer Persönlichkeit, wobei scheinbar unwichtige und unbedeutende Begebenheiten für sie von Bedeutung sind, da sie aus ihnen heraus größere Zusammenhänge zu begreifen sucht. Hells Vorliebe für den Verfall, für das Krankhafte und Hässliche ist keine Attitüde, vielmehr manifestiert sich darin ihre Erfahrung und ein autotherapeutisches Verfahren. Dadurch erhält die neue Identitätsarbeit eine zusätzliche Verankerung in einer neuen Vergangenheitsbewältigung. Sie definiert Schicksal als alles, was auf sie zukommt und was sie zur Verteidigung und Selbstbehauptung aufruft.

Andere Gewaltformen präsentiert Ingo Schramm, wobei er Gewalt nicht ausschließlich aus der Perspektive eines Opfers schildert. Josefs Denken kreist ständig um die Frage, welche Formen seine Destruktivität noch annehmen könne. Seine Kinder werden verängstigt, beschimpft, verspottet, erniedrigt, geschlagen und terrorisiert, was seine philosophisch veranlagte Tochter Janni eines Tages feststellen lässt, dass

die Gesetze des Wachstums die Gesetze des Untergangs sind. Die Welt ist ein Hefekuchen, der von den eigenen Zutaten aufgebläht wird und zusammenbricht, während der Bäcker sich eben die Zinsen seines Gewinns hochrechnet, denn er will von etwas beeindruckt sein. [...] Am Tagesende räumt der Bäcker auf, wirft die nicht abgesetzten Kuchen in die Mülltonne, wo sich die Ratten hineinstehlen und in Besitz nehmen, was der Mensch verschmähte. Sie haben andre Kriterien, sie leben nach anderem Maßstab, der ist uns nicht zu ermessen. (SCHRAMM 1996:257)

Dieser Mann ist von starker Aggressivität geprägt, auch wenn die Außenwelt diese kaum wahrnimmt; er scheint nahezu eine ,Lizenz zur Folterung 'seiner Familienmitglieder zu haben. Wie ein Henker foltert er seine Opfer, wobei er diese ,Experimente' mit seiner wissenschaftlichen Tätigkeit erklärt: 
Aleksandra Chylewska-Tölle

Der Wille hatte ihn aufgestellt, im Unsichtbaren das Große zu wirken. Die Umgestaltung der Welt erwies sich als langwierig und kompliziert. Wer im Offenen wirkte, dem stand die Öffentlichkeit entgegen. Dem Verborgenen aber konnte nichts widerstehen. (SCHRAMM 1996:351)

Es wäre zu fragen, ob der Grund für Josefs Taten, die von starker Zerstörungslust zeugen, nicht in erster Linie darin zu suchen ist, dass er seine brutalen ,Erziehungsmethoden“ nicht als Misshandlung, sondern als Züchtigungsrecht bezeichnet. Seine Macht dient ihm außerdem dazu, sein inneres Gleichgewicht herzustellen, das aufgrund seiner Gewalterfahrungen in der Kindheit immerzu bedroht zu sein scheint. Somit besteht das Paradox dieser Gewalttätigkeit darin, dass sie gerade dem grundlegenden Bedürfnis nach Gleichgewicht zuwiderläuft. Schramm enthält sich jeglichen Urteils über seinen Protagonisten, er rechtfertigt ihn jedoch keineswegs. Ganz im Gegenteil: Er betrachtet Gewalt nicht in Kategorien einer natürlichen und historisch bedingten Gegebenheit, sondern als Produkt menschlicher Konstitution. Diese Überzeugung illustriert Staffels Roman in noch stärkerem Maße. Für ihn ist die mit Aggressivität und Hass beginnende Gewalt nicht das Resultat einer geschichtlichen Veränderung, sondern der Mensch selbst wird zum Verursacher der Apokalypse.

Die Misshandlungen führen bei Pareis Titelfigur und Schramms jungen Protagonisten zu psychosozialen Folgen und manifestieren sich sowohl im Wohnortwechsel, in der Kündigung des Arbeitsplatzes und im Abbrechen der Kontakte zur Außenwelt (Hell), in psychischer Schwäche, vermindertem Selbstwertgefühl (Karl) und in der Rationalisierung der erlebten Wirklichkeit durch Philosophieren (Janni). Diese Abwehrmechanismen erscheinen als Selbstbetrug, da sie dem Geschehenen den Anschein logischer Begründung geben. Der Selbstbetrug stützt sich auf ,objektive Bedingungen', die das eigene Versagen rechtfertigen und eine positive Haltung gegenüber der erfahrenen Gewalt ermöglichen. Den von Schramm und Staffel dargestellten Gewaltopfern bleiben kaum Möglichkeiten der Gegenwehr, nicht zuletzt weil die Bedrohung plötzlich und unerwartet auftaucht - was im Übrigen ganz den Vorstellungen Josefs und der nicht näher geschilderten Gewalttäter im Terrordrom entspricht. In Staffels Roman begegnet dem Leser auch eine Form der häuslichen Tyrannei: Die Schwere der erlebten Gewalt im Eheleben kann Toms Frau Anna nicht mehr ertragen. Nachdem sie jahrelang körperliche Misshandlungen geduldet hat, beschließt sie endlich nach einer erneuten Gewaltattacke, ihren Mann zu verlassen. Mit diesem Schritt enttabuisiert sie diese Form häuslicher Gewalt. In allen drei Romanen ist unabhängig von der Schwere des Verbrechens vor allem die männliche 
Gewaltbereitschaft offenkundig. Den Frauen bleibt meist die Rolle des Opfers, wenn sich auch einige (dies betrifft u. a. Toms Sekretärin und zugleich Geliebte Anette) unter dem gewaltfördernden Einfluss in dunkle Handlungen verstricken lassen. Die betroffenen Menschen sind dabei unfähig, ihre leidvollen Erfahrungen zu verbalisieren, da Gewalt ein Ausdruck der Sprachlosigkeit ist, was am deutlichsten am Beispiel der Protagonistin Hell präsentiert wird. Mit anderen Worten: Die erfahrene Ungerechtigkeit zerstört die Kommunikation. Auch Staffel belegt diesen Mechanismus anhand der von ihm skizzierten Berliner Gesellschaft, die sich das Kommunizieren abgewöhnt hat. Ironisch wird dies mit den von Tom moderierten Talkshows gezeigt, in denen ohne jedwedes Verständnis und Einfühlungsvermögen gegenüber dem Gesprächspartner nur ,gequatscht ‘ wird.

Im Berlin der Nachwendezeit kristallisieren sich die Erlebnisse, Erfahrungen und Wünsche von Pareis, Schramms und Staffels Protagonisten. Obwohl in der Schattenboxerin der Handlungszeitraum zum Teil in die unmittelbare Vorwendezeit zurückreicht (Hell wurde im Sommer 1989 vergewaltigt), wird die Aufmerksamkeit des Lesers insbesondere auf den Zeitraum nach dem Mauerfall gelenkt. Die Ich-Erzählerin bewegt sich zwischen Vergangenheit und Gegenwart, die ineinanderfließen und den Eindruck einer Einheit bilden. Bei allen hier genannten Autoren ist die Wahl des Handlungsortes sehr bewusst erfolgt. In Berlin verbinden sich Geschichte und Umbruch, Majestät und Untergang, Ost und West besonders intensiv. Damit ist ein wesentlicher Aspekt angesprochen: Das Leben der Protagonisten bewegt sich zwischen zwei Polen: Stillstand und Bewegung, Beharrung und Veränderung. Indem dramatische zeitgeschichtliche Ereignisse (bei Parei sind Reminiszenzen an den Mauerfall und bei Schramm an die deutsche Wiedervereinigung zu finden) mit Familienschicksalen verbunden werden, knüpfen die Autoren deutlich an die Tradition des Großstadtromans an, deren Vorbild Döblins Berlin Alexanderplatz ist. Die Westdeutschen Parei und Staffel scheinen jedoch die Berlin-Mode in der Literatur gewissermaßen zu parodieren, da ihre Romane anti-urbane und vielmehr, unmenschliche ' Züge der Stadt aufdecken (DAHLKE 2002:460-462). So wird Berlin zum Abbild der Seelenlandschaft einiger von Gewalt direkt betroffener Protagonisten. Die Perspektive der beiden Verfasser ist auch dadurch gekennzeichnet, dass sie zwar realitätsnahe Einblicke in den Berliner Alltag gewähren, jedoch ohne Berücksichtigung der durch die Wende ausgelösten Verlusterfahrungen und ohne eine Auseinandersetzung mit den sozialistischen Utopien, wie das hingegen bei dem Ostdeutschen Schramm - beabsichtigt oder nicht - festzustellen ist (STENGER 1997:45). Keiner von ihnen ist bestrebt, die touristischen Anziehungspunkte 
Aleksandra Chylewska-Tölle

der Stadt hervorzuheben. In Pareis Roman wird der Gegensatz zwischen dem Zentrum (Berlin-Mitte, Kreuzberg, Neukölln) und den Stadtperipherien (Treptow, Lübars, Schöneweide) in der Überzeugung betont, dass sich „Berlin nun von den Rändern her neu erschließen müsse“ (MENsING 1999:22). Die von der Autorin geschilderte deprimierende Stimmung der deutschen Hauptstadt ist auch bei Schramm zu finden, wobei Letzterer den Prozess der stetigen Veränderung noch intensiver hervorhebt. Und was bei ihm auf den ersten Blick wie ein „ostalgischer Anfall“ aussieht, ist vielmehr der Ausdruck seiner Ratlosigkeit gegenüber den geschichtlichen Umbrüchen (GABLER 2000:85). In Terrordrom wird der Leser mit Berlins Zentrum als Ruinenlandschaft, entstanden infolge terroristischer Anschläge, und als SchneeDschungel konfrontiert, womit zugleich der psychische Zustand vieler Bewohner der Stadt seinen Ausdruck findet. Staffel verzichtet ebenfalls auf die Darstellung des urbanen Lebens in der deutschen Metropole. Stattdessen entstehen in seinem Roman Bilder von abgegrenzten Lebensräumen, d.h. von einzelnen Straßen, Kneipen, Gebäuden. Alle Autoren schildern dabei Berlin als Ort des Verbrechens und der tiefsten äußeren und inneren Verwahrlosung.

Nihilistische Verunsicherung und Verzweiflung, heilloser Werterelativismus als Abwehrstrategie, Gewalt gegen Minderheiten, Krawalle zwischen Fußballfans, der Amoklauf der Massen im Terrordrom, Überfälle, Ausbeutung, ausgeprägter Egoismus, Ausländerhass, die Auflösung der traditionellen Bindungen, der Zerfall überkommener Werte - all dies ist reichlich in den drei behandelten Romanen zu finden, die im Übrigen eine gewisse Exemplarizität beanspruchen können. An ihnen lässt sich nämlich zeigen, dass die Verbindung des Topos Berlin mit der Gewaltthematik kein marginales Phänomen ist, sondern viele Texte kennzeichnet. Einerseits übernimmt Gewalt bestimmte Funktionen innerhalb einer Stadtkonstruktion, andererseits offeriert das Stadtgefüge manche Symbolfunktionen für Gewaltkonstellationen. Diese werden hier als Mittel der Symbolisierung (Parei), der Destruktion, Prüfung, Bewährung (Schramm), der scheiternden Kommunikationsversuche und der Dekonstruktion (Staffel) erprobt. Auf jede Gewaltanwendung folgen deren Konsequenzen, was bedeutet, dass es den Gewalttätern unmöglich ist, der Verantwortung für ihre Handlungen zu entkommen. Somit wird deutlich, dass Gewalt Teil der Zivilisation ist, die ihre hehren Werte und Ideale am Übergang zum 21. Jhd. laut preist. Dies hängt u. a. damit zusammen, dass der Mensch von seiner Natur her - was Schramm und Staffel deutlich zeigen ein gewalttätiges Wesen ist. Eine mit Gewalt beladene Vergangenheit und auf den ersten Blick harmlose, kleine Charakterschwächen können einen enormen Hass auslösen. Der Mensch unterscheidet sich vom Tier vor allem 
dadurch, dass seine Handlungen nicht ausschließlich aus dem Willen des Überlebens, sondern auch des Wohllebens resultieren. Und dennoch tritt die moralische Bewertung der Gewaltdarstellung in den drei Romanen eher in den Hintergrund. Die Autoren konzentrieren sich vielmehr auf die ästhetische Kodierung von Gewaltphänomenen und auf deren Miteinbeziehung in den Kulturdiskurs der Zeit.

Walter Benjamins Frage nach der Möglichkeit einer Rechtfertigung von Gewalt als Mittel zu gerechten Zwecken (BENJAMIN 1965:29) wird von den drei Autoren jedoch entschieden verneint. Gewalt mag zwar - wenn auch lediglich indirekt - eine positive Entwicklung des Menschen befördern bzw. seine Entwicklung positiv steuern, dies ist jedoch selten der Fall. Im Übrigen ist nur bei Staffel ein solcher Fall am Beispiel von Felix dargestellt, der seine misshandelte Mutter unterstützt. Gezeigt werden so gewissermaßen gewaltfreie ,Inseln“, die in einem Meer bitterer Erfahrungen bestehen können. Hell, die Kung-Fu beherrscht, befreit den Besitzer ihrer Stammkneipe von Gelderpressern. Karl träumt von einer besseren, gewaltlosen Welt. Als sich seine Wünsche als utopisch erweisen, beschließt er, seine Halbschwester Janni zu schützen. Das Bedürfnis nach innerer Freiheit wird bei den Protagonisten von der Sehnsucht nach einer Verbesserung der Welt begleitet. Zynisch klingen nur die Worte des Erzählers bezüglich einer neu ausgebrochenen Hysterie im Terrordrom: „Und jetzt flippen alle Freaks aus. Die große Welle. Es knallt und kracht überall. Wir spielen Krieg und rennen irgendwas hinterher. Und dieses Wir, das es sein soll, ist der totale Schwachsinn. Und keiner merkt das.“ (STAFFEL 1998:184) Trotz dieses apokalyptischen Beigeschmacks der Geschichte ist doch auch in ihr noch eine tiefverborgene Hoffnung auf einen Neubeginn zu finden.

\section{Literatur}

ARENDT, HANNAH (1971): Macht und Gewalt. München.

Benjamin, WAlter (1965): Zur Kritik der Gewalt und andere Aufsätze. Frankfurt (M.).

DAHLKe, BIRGIT (2002): Berlin-Frontstadt, Mauerstadt, Metropole? Zum literarischen Zusammenhang von Stadtbild und Gesellschaftsutopie. In: STILLMARK, HANSCHRISTIAN / LEHKER, CHRISTOPH (eds.): Rückblicke auf die Literatur der DDR. Amsterdam/New York, 455-472.

GABLER, WolfGANG (2000): Der Wenderoman als neues literarisches Genre. Thesen. In: GABler, WolfGANG / WeRZ, Nikolaus (eds.): Zeiten - Wende - Wendeliteratur. Umrisse. Weimar/Rostock, 70-92. 
Aleksandra Chylewska-Tölle

Heitmeyer, Wilhelm / Schröttle, Monika (eds.) (2006): Gewalt. Beschreibungen, Analysen, Prävention. Bonn.

IMBUSCH, PETER (2006): Terrorismus - ideologische Spannweite, Charakteristiken, historische Ursachen. Fachwissenschaftliche Analyse. In: HEITMEYER / SCHRÖTTLE, 487-510.

KoRmann, Julia (1999): Literatur und Wende. Ostdeutsche Autorinnen und Autoren nach 1989. Wiesbaden.

Mensing, KolJa (1999): Rezensionsnotiz zu: Die Tageszeitung, 13.11.1999.

Müller, Ursula / Schröttle, MoniKa (2006): Gewalt gegen Frauen in Deutschland - Ausmaß, Ursachen und Folgen. Fachwissenschaftliche Analyse. In: HeITMEYER / SCHRÖTTLE, 77-97.

PAREI, INKA (2001): Die Schattenboxerin. Frankfurt (M.).

REEMTSMA, JAN PhILIPP (2008): Vertrauen und Gewalt. Versuch über eine besondere Konstellation der Moderne. Hamburg.

Schramm, Ingo (1996): Fitchers Blau. Poetischer Roman. Berlin.

STAFFEL, TiM (1998): Terrordrom. Zürich.

STENGER, Cordula (1997): „Stolz und Vorurteil“. Junge deutsche Dichter über deutsche Dinge nach der Wende 1989. Bremen.

STILLMARK, HANS-CHRISTIAN / LEHKER, CHRISTOPH (2002): Rückblicke auf die Literatur der DDR. Amsterdam/New York.

Witorska, Alicja (2005): Co to jest agresja? Studium semantyczne. [Was ist Aggressivität? Ein semantisches Studium]. In: DąBRowsKa, ANNA / NowaKowsKA, AlicJA (eds.): Język a kultura. Życzliwość i agresja w języku i kulturze. Wrocław. 